\title{
Congestive heart failure during induction with anthracycline-based therapy in patients with acute promyelocytic leukemia
}

\author{
Vamsi Kota, MD, ${ }^{a}$ Amber Clemmons, PharmD, ${ }^{\mathrm{b}, \mathrm{c}}$ Arati Chand, MD, ${ }^{\mathrm{b}}$ Josh Simmons, MD, \\ Joshua Mansour, MD, ${ }^{c}$ Ravindra Kolhe, $\mathrm{MD},{ }^{\mathrm{d}}$ and Anand Jillella, $\mathrm{MD}^{\mathrm{a}}$
}

Winship Cancer Institute of Emory University, Atlanta, Georgia; 'beorgia Regents University Cancer Center, Augusta, Georgia; 'University of Georgia College of Pharmacy, Athens, Georgia; and Departments of 'Medicine and Pathology, Georgia Regents University

\begin{abstract}
Background Acute promyelocytic leukemia (APL) is a highly curable malignancy. However, 30\% of patients die during therapy induction from bleeding, differentiation syndrome (DS), and/or infection. Recommendations suggest that congestive heart failure (CHF) is a presenting feature of DS.

Objective To assess the incidence of CHF during induction in patients with APL.

Methods A retrospective chart review was performed of patients diagnosed with APL from December 2004 to July 2013 and managed at Georgia Regents University Cancer Center. Baseline and follow-up ejection fractions (EF) were recorded and patients with a drop in EF during the induction period were evaluated.

Results Of the 40 evaluable patients, 37 received idarubicin-based chemotherapy. 16 of the 37 patients had a repeat ECHO for suspected cardiomyopathy, and 6 of the 16 patients (37.5\%) demonstrated a decrease in EF (absolute drop, 10\%-35\%). The cardiac function recovered completely in 4 patients and partially in 1 patient. Gender, history of hypertension, and body mass index did not seem to correlate with incidence of CHF.

Limitations The patient population is very small given the rarity of the disease. Present practice patterns do not routinely address $\mathrm{CHF}$ in the differential diagnosis.

Conclusions Patients with APL are at risk for cardiac toxicity for a number of reasons, including cytokine storm and inflammatory state, use of anthracyclines, and DS. The clinical presentation of DS most commonly involves dyspnea and fluid retention, which are also symptoms of heart failure. Prompt cardiac evaluation should be undertaken to rule out CHF in APL patients who are going to receive an anthracyclinebased therapy, because early intervention may result in an improved outcome.
\end{abstract}

A cute promyelocytic leukemia (APL) is a rare and distinct subtype of acute myeloid leukemia that is characterized by the $t(15 ; 17)$ translocation resulting in the fusion of the promyelocytic leukemia (PML) gene on chromosome 15 to the retinoic acid receptor alpha gene on chromosome 17. ${ }^{1}$ Targeted treatment with all-trans retinoic acid (ATRA) can result in the differentiation of blasts and has been shown to improve outcome and reduce relapse rates. Although APL is considered a highly curable malignancy - it has a cure rate of up to $90 \%$ - findings from co-operative group trials and population-based studies have shown survival rates of $65 \%-70 \%$, with up to $30 \%$ early mortality in the 30 days after diagnosis. ${ }^{2,3}$ The most frequent causes of early death are bleeding, infection, and differentiation syndrome (DS). ${ }^{4}$ Differentiation syndrome, which was formerly known as retinoic acid or ATRA syndrome), is a potentially fatal clinical syndrome that occurs most often in the first 3 weeks of treatment with ATRA or arsenic trioxide and is associated with a mortality of $2 \%-27 \% .^{5} \mathrm{DS}$ is generally characterized by increases in the white blood cell count, weight gain, renal failure, dyspnea, pleural or pericardial effusion, pulmonary infiltrates, and fever. ${ }^{4}$ The wide spectrum in presenting symptoms can lead to a delay in diagnosis and possibly a deleterious effect on survival. The European Leukemia Net recommendations suggest that congestive heart failure (CHF) is one of the presenting features of DS, however, most of the reports on cardiac abnormalities focus on pericardial effusion. ${ }^{6}$ The exact incidence of CHF during the induction period has not been well described in the literature. Cardiac stunning, which is a transient decrease in cardiac function because of sudden cardiac insult, is only briefly reported. ${ }^{7}$ This might be a result of cytokine storm attributable to tumor lysis in addition to being part of the DS. Although late onset of $\mathrm{CHF}$ has been reported in patients with $\mathrm{APL},{ }^{8}$ herein we report the incidence of $\mathrm{CHF}$ in patients during induction for APL with ATRA.

Accepted for publication July 1, 2014. Correspondence: Vamsi Kota, MD; vkota@emory.edu. Disclosures: The authors have no disclosures. JCSO 2014;12:390-393. C2014 Frontline Medical Communications. DOI 10.12788/jcso.0084. 


\section{Methods}

We performed a retrospective chart review to identify patients diagnosed with APL between December 1, 2004 and July 31, 2013 at Georgia Regents Medical Center and the surrounding referring centers we help manage. A total of 41 consecutive patients were identified, all of whom had data for a baseline ejection fraction. One patient refused treatment and was excluded, leaving a total of 40 patients in the study group. IRB approval and patient consents were obtained to access clinical data.

Demographic information (age, sex, induction chemotherapy, history of hypertension, body mass index) was obtained from the charts. Baseline and follow-up ejection fractions $(\mathrm{EF})$ were recorded by echocardiogram $(\mathrm{ECHO})$ or nuclear medicine (MUGA) scan results as documented in the medical records. We analyzed patients with a significant drop in EF, as defined by a reduction in EF by at least $10 \%$. Future echocardiogram or MUGA scan results were also recorded if they were available for those patients with a reduction in EF.

Time to onset of differentiation syndrome was recorded as number of days between diagnosis and first documentation of differentiation syndrome. Survival was recorded as number of days from diagnosis to documented death or censored at last known follow-up.

\section{Results}

Of the 40 patients, 21 (52.5\%) were men, and the median age was 54 years (range, 19-81; mean, 51.7 years). Most of the patients (35) received induction therapy with idarubicin plus ATRA, 3 patients received arsenic trioxide plus ATRA, and 2 patients received 7+3 (cytarabine plus daunorubicin) and ATRA. In all, 7 patients (17.5\%) died during the induction period (range, days 5-34).

Sixteen patients (40\%) had an ECHO to assess the ejection fraction during the first 30 days of treatment for suspected cardiomyopathy. Of those patients, 6 (37.5\%) had a documented reduction in EF (Table 1). The age range for the 6 patients was $42-81$ years (median, 55), half of them were male, and all had received idarubicin as part of their induction treatment (idarubicin plus ATRA). The absolute reduction in $\mathrm{EF}$ was 10\%-30\%; 1 of the 6 patients had elevation in troponin levels. Four of the 6 patients (67\%) had a diagnosis of significant differentiation syndrome. Of the 6 patients with a documented drop in EF, 5 had a followup ECHO to assess cardiac function. Four patients had complete recovery of $\mathrm{EF}$, and 1 patient recovered the EF to baseline of 45\%. Two of the 6 patients with a drop in EF died during induction, with 1 death at day 18 , and another at day 33 .

The remaining 10 patients who had a repeat $\mathrm{ECHO}$ but did not have a reduction in EF had similar characteristics (Table 2). Their median age was 62 years (range, 19-78),
9 of the 10 patients had received ATRA plus idarubicin induction, 6 were men, and 7 had differentiation syndrome. A history of hypertension was documented in 4 of the 6 patients (67\%) with a drop in EF compared with 6 of the 10 patients (60\%) who did not have a drop EF. Patients with a drop in EF had a median body mass index of 28.8 (range, 20.4-38.2; mean, 28.7) , compared with 34.5 (range, 26.2-52.6; mean, 34.5) for the patients without a drop in EF. The 24 patients who did not have a repeat ECHO did have baseline characteristics that differed significantly from the patients in whom ECHO was done (Table 3). In the group that did not have a repeat $\mathrm{ECHO}$, half of the patients were men, $88 \%$ received ATRA plus idarubicin, and the median age was 50 years (range, 20-77). However, only $42 \%$ had differentiation syndrome.

For the 21 patients with documented differentiation syndrome, the median time to onset of DS was 10 days (range, 1-23; mean, 10.8). The median overall survival of the entire population was 430 days (range, 5-2,892; mean, 886). The median overall survival of the 6 patients with a documented drop in EF was 744 days (range, 18-2,588; mean, 1,080), compared with 147 days (range, 17-2,270; mean, 606) for the 10 patients who had a repeat ECHO and no change in EF.

\section{Discussion}

Advances in the treatment of APL with the use of ATRA and arsenic trioxide, either alone or in combination with traditional chemotherapy, have substantially improved clinical outcomes for this rare malignancy. ${ }^{9}$ However, survival for this group of patients also depends on appropriate and aggressive use of supportive care to prevent fatal complications from the disease and its therapy. ${ }^{3}$ Specifically, the prompt assessment and management of differentiation syndrome is crucial. The most common presentation of DS is respiratory distress, weight gain, and fluid retention. ${ }^{10}$ These are also common clinical symptoms of heart failure, and it is likely that some of these symptoms are attributed to DS without appropriate evaluation of cardiac problems.

Anthracycline-induced cardiac injury has been well described. It most commonly occurs after repeated doses of anthracycline-based therapy and the incidence correlates with the lifetime dose administered. ${ }^{12}$ The pathophysiology of cardiac injury seems to be caused by the generation of free radicals as a result of repeated injury to cardiac myocytes. ${ }^{11}$ Most of the available literature on cardiotoxicity comes from the evaluation of patients with breast cancer. The described incidence of chronic progressive cardiotoxicity at doses under $300 \mathrm{mg} / \mathrm{m}^{2}$ of doxorubicin is less than $3 \%{ }^{12}$ Although cardiotoxicity has been described after a single dose of anthracyclines, it rarely leads to serious deterioration. ${ }^{12}$

In the present study, of the 40 patients who were treated, 
TABLE I Patients with reduction in ejection fraction

\begin{tabular}{|c|c|c|c|c|c|c|c|c|c|c|c|c|}
\hline $\begin{array}{l}\text { Patient } \\
\text { ID no. }\end{array}$ & Age, y & Sex & BMI & History & Treatment & $\begin{array}{c}\text { Baseline } \\
\text { EF, \% }\end{array}$ & $\begin{array}{c}\text { Repeat } \\
\text { EF, \% }\end{array}$ & $\begin{array}{l}\% \text { drop } \\
\text { in EF }\end{array}$ & $\begin{array}{c}\text { Recovery of } \\
\text { EF, \% }\end{array}$ & DS & $\begin{array}{l}\text { Time } \\
\text { of DS } \\
\text { onset, d }\end{array}$ & $\begin{array}{c}\text { Survival, } \\
\text { d }\end{array}$ \\
\hline 2 & 56 & M & 30.2 & Y & ATRA+IDA & 55 & 25 & 30 & $55 \%$ & $\mathrm{~N}$ & No DSa & 2,354 \\
\hline 1 & 56 & $\mathrm{~F}$ & 20.4 & Y & ATRA+IDA & 46 & 20 & 26 & 45 & $Y$ & 8 & 2,588 \\
\hline 23 & 54 & $M$ & 27.4 & Y & ATRA+IDA & 60 & 45 & 15 & 55 & $Y$ & 10 & 1,172 \\
\hline 39 & 42 & $\mathrm{~F}$ & 34.9 & $\mathrm{~N}$ & ATRA+IDA & 65 & 45 & 20 & 50 & $Y$ & 5 & 18 \\
\hline
\end{tabular}

TABLE 2 Patients without reduction in ejection fraction

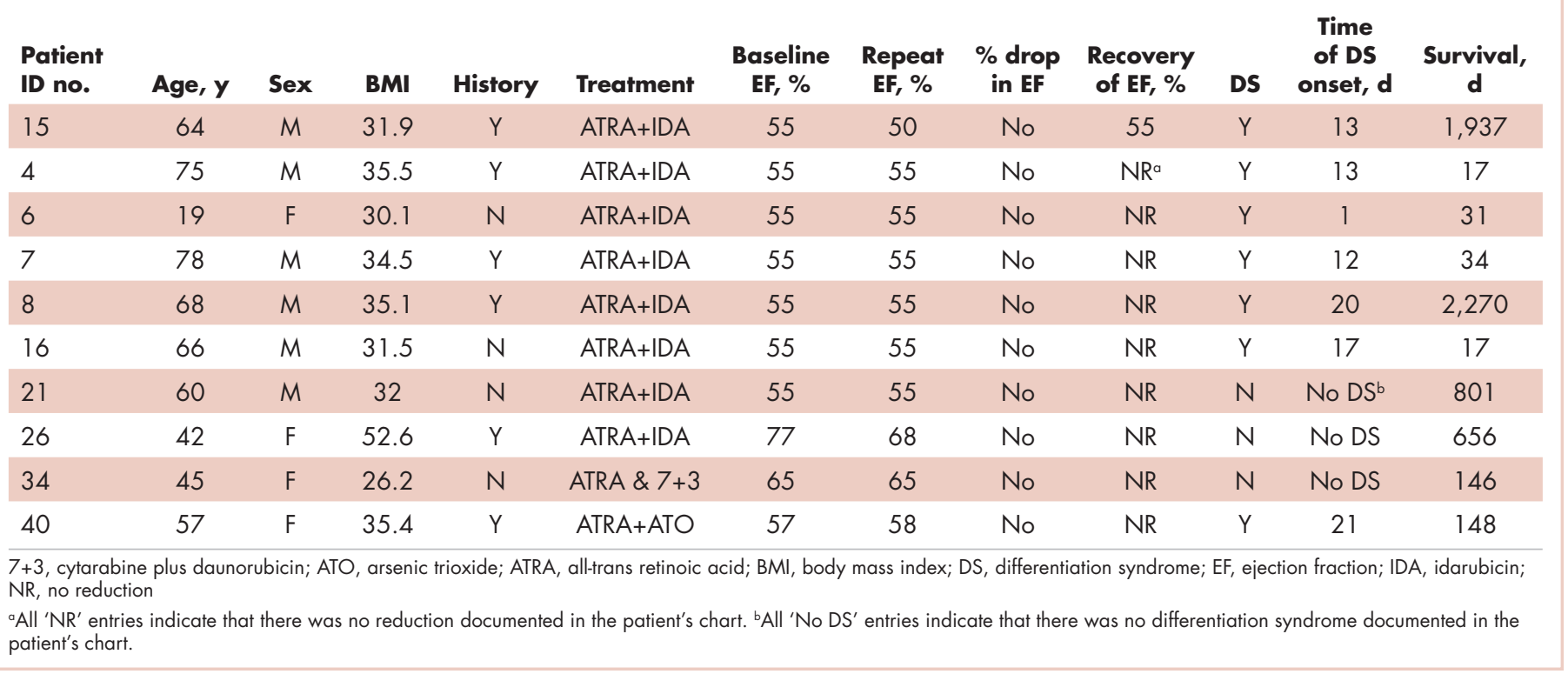

we have shown a reduction in EF in 6 of 16 patients who had an ECHO to assess the ejection fraction during the first 30 days of treatment for suspected cardiomyopathy. A follow-up cardiac evaluation was not undertaken for all of the patients with differentiation syndrome, the symptoms of which overlap with those of heart failure. The actual incidence of heart failure might be even higher if thoroughly evaluated. A very small number of patients received arsenic during induction and given this low number, it may be difficult to draw any conclusions regarding the incidence of heart failure in arsenic-treated patients. None of the characteristics evaluated (body mass index, cardiac risk factors, age, or sex) seemed to correlate with incidence of heart failure. Patients who were treated with anthracyclinebased consolidation regimens may receive additional cycles of anthracycline resulting in further deterioration of cardiac function. This has to be carefully considered, and car- diac function should be closely monitored in this highly curable disease to minimize major organ system toxicity and morbidity.

\section{Conclusions}

Heart failure can occur in patients undergoing induction therapy for acute promyelocytic leukemia, especially if anthracycline-based regimens are used. Patients who have signs or symptoms of heart failure, with or without other evidence of differentiation syndrome, should have their cardiac function re-evaluated. This might have immediate and long-term treatment implications. We acknowledge that this is in a small series of patients and even fewer number of patients with follow-up echocardiograms done. It is not common practice to get echocardiograms in patients who exhibit signs of differentiation syndrome. These results have to be validated further in a larger cohort of patients 
TABLE 3 Patients without ejection fraction re-evaluation

\begin{tabular}{|c|c|c|c|c|c|c|c|}
\hline Patient ID no. & Age, y & Sex & Treatment & Baseline EF, \% & DS & Time of DS onset, $d$ & Survival, d \\
\hline 3 & 49 & $\mathrm{~F}$ & ATRA+IDA & 80 & Y & 2 & 11 \\
\hline 9 & 57 & M & ATRA+IDA & 55 & Y & 15 & 2,255 \\
\hline 10 & 66 & M & ATRA+IDA & 55 & Y & 2 & 2,263 \\
\hline 11 & 39 & M & ATRA+IDA & 55 & $\mathrm{~N}$ & No DSa & 2,114 \\
\hline 12 & 77 & M & ATRA+IDA & 55 & $N$ & No DS & 2,093 \\
\hline 13 & 50 & $\mathrm{~F}$ & ATRA+IDA & 55 & Y & 20 & 2,087 \\
\hline 14 & 53 & M & ATRA+IDA & 55 & $N$ & No DS & 2,011 \\
\hline 17 & 63 & $\mathrm{~F}$ & ATRA+IDA & 55 & Y & 2 & 5 \\
\hline 18 & 62 & $\mathrm{~F}$ & ATRA+IDA & 65 & $\mathrm{~N}$ & No DS & 2,892 \\
\hline 19 & 30 & M & ATRA+IDA & 55 & $N$ & No DS & 1,051 \\
\hline 20 & 31 & $\mathrm{~F}$ & ATRA+IDA & 55 & Y & 1 & 959 \\
\hline 22 & 52 & $\mathrm{~F}$ & ATRA+IDA & 55 & Y & 3 & 816 \\
\hline 24 & 38 & M & ATRA+IDA & 55 & $N$ & No DS & 951 \\
\hline 25 & 54 & M & ATRA+IDA & 65 & $\mathrm{~N}$ & No DS & 749 \\
\hline 27 & 42 & M & ATRA+IDA & 55 & $\mathrm{~N}$ & No DS & 437 \\
\hline 28 & 20 & M & ATRA+IDA & 65 & $N$ & No DS & 423 \\
\hline 29 & 54 & $\mathrm{~F}$ & ATRA+IDA & 55 & $\mathrm{~N}$ & No DS & 407 \\
\hline 30 & 58 & $\mathrm{~F}$ & ATRA+IDA & 65 & $\mathrm{~N}$ & No DS & 336 \\
\hline 32 & 23 & M & ATRA+IDA & 65 & Y & 21 & 300 \\
\hline 33 & 43 & $\mathrm{~F}$ & ATRA+IDA & 55 & $\mathrm{~N}$ & No DS & 112 \\
\hline 35 & 69 & $\mathrm{~F}$ & ATRA \& 7+3 & 65 & $\mathrm{~N}$ & No DS & 129 \\
\hline 36 & 44 & M & ATRA+ATO & 65 & Y & 7 & 153 \\
\hline 37 & 44 & $\mathrm{~F}$ & ATRA+ATO & 55 & Y & 23 & 175 \\
\hline 38 & 33 & $\mathrm{~F}$ & ATRA+IDA & 66 & $N$ & No DS & 166 \\
\hline
\end{tabular}

7+3, cytarabine plus daunorubicin; ATO, arsenic trioxide; ATRA, all-trans retinoic acid; DS, differentiation syndrome; EF, ejection fraction; IDA, idarubicin aAll 'No DS' entries indicate that there was no differentiation syndrome documented in the patient's chart.

and also need a lower threshold to repeat an echocardiogram with the symptoms of differentiation syndrome.

\section{References}

1. Tallman MS, Altman JK. How I treat acute promyelocytic leukemia. Blood. 2009;114:5126-5135.

2. Lehmann S, Ravn A, Carlsson L, et al. Continuing high early death rate in acute promyelocytic leukemia: a population-based report from the Swedish Adult Acute Leukemia Registry. Leukemia. 2011;25:1128-1134.

3. Jacomo RH, Melo RA, Souto FR, et al. Clinical features and outcomes of 134 Brazilians with acute promyelocytic leukemia who received ATRA and anthracyclines. Haematologica. 2007;92:14311432.

4. De Botton S, Dombret H, Sanz M, et al. Incidence, clinical features, and outcome of all trans-retinoic acid syndrome in 413 cases of newly diagnosed acute promyelocytic leukemia. The European APL Group. Blood. 1998;92:2712-2718.

5. Montesinos P, Bergua JM, Vellenga E, et al. Differentiation syndrome in patients with acute promyelocytic leukemia treated with all-trans retinoic acid and anthracycline chemotherapy: characteristics, outcome, and prognostic factors. Blood. 2009;113:775-783.

6. Sanz MA, Grimwade D, Tallman MS, et al. Management of acute promyelocytic leukemia: recommendations from an expert panel on behalf of the European LeukemiaNet. Blood. 2009;113:1875-1891.

7. De Santis GC, Madeira MI, de Oliveira LC, Falcao RP, Rego EM. Cardiac stunning as a manifestation of ATRA differentiation syndrome in acute promyelocytic leukemia. Med Oncol. 2012;29:248250.

8. Thomas X, Le QH, Fiere D. Anthracycline-related toxicity requiring cardiac transplantation in long-term disease-free survivors with acute promyelocytic leukemia. Ann Hematol. 2002;81:504-507.

9. Lo-Coco F, Avvisati G, Vignetti M, et al. Retinoic acid and arsenic trioxide for acute promyelocytic leukemia. N Engl J Med. 2013;369:111-121.

10. Tallman MS. Differentiating therapy with all-trans retinoic acid in acute myeloid leukemia. Leukemia. 1996;10(Suppl 1):S12-5.

11. Rafiyath SM, Rasul M, Lee B, Wei G, Lamba G, Liu D. Comparison of safety and toxicity of liposomal doxorubicin vs. conventional anthracyclines: a meta-analysis. Exp Hematol Oncol. 2012;1:10.

12. Von Hoff DD, Layard MW, Basa P, Davis HL, Jr., Von Hoff AL, Rozencweig M, et al. Risk factors for doxorubicin-induced congestive heart failure. Ann Intern Med. 1979;91:710-717. 